Ergod. Th. \& Dynam. Sys. (1988), 8, 637-650

Printed in Great Britain

\title{
Dynamics of the geodesic flow of a foliation
}

\author{
PAWEL G. WALCZAK
}

University of Łódź, Institute of Mathematics, Banacha 22, PL 90238 Łódź, Poland

(Received 15 July 1987 and revised 8 January 1988)

\begin{abstract}
The geodesic flow of a foliated Riemannian manifold $(M, F)$ is studied. The invariance of some smooth measure is established under some geometrical conditions on $F$. The Lyapunov exponents and the entropy of this flow are estimated. As an application, the non-existence of foliations with 'short' second fundamental tensors is obtained on compact negatively curved manifolds.
\end{abstract}

\section{Introduction}

The geodesic flows of leaves of a foliated Riemannian manifold $(M, F)$ glue together with a flow on the unit tangent bundle of $F$. This is the geodesic flow of $F$, the main object of our interest here.

We have at least two reasons for studying this flow.

First, the geodesic flow of a Riemannian manifold $M$ has been intensively studied during the last few years (see [A, BBE, BBS, E, FM, Ma1, Ma2, K, OS] and many others) and it has been established that the dynamical properties of this flow reflect the geometry of $M$. So, we may expect that the geodesic flow of a foliation could become a good tool in the study of the geometry of $F$.

Second, the last few years have seen some attempts concerning the dynamical theory of foliations. The idea is to adapt some notions and methods of the theory of dynamical systems to the theory of foliations. In this direction, we have, for example, studies of invariant measures and some ergodic properties of foliations [Ga, PI] or an attempt [GLW] made at defining and studying the entropy of a foliation.

Also, let us note that the dynamics of the geodesic flow was used successfully [Z] to study totally geodesic foliations of locally symmetric spaces.

In this paper, after giving some preliminary information, we begin with varying geodesics on leaves among such geodesics. This leads to a suitable notion of Jacobi fields $(\$ 2)$. While studying natural Riemannian metrics on the unit tangent bundle of a foliation we ask when the Riemannian volume is preserved by our flow ( $\$ 3$ ). Jacobi fields are used to estimate Lyapunov exponents $(\$ 4)$ and the entropy $(\$ 5)$ of this flow. Using these estimations in $\S 6$, we prove that there are no totally geodesic foliations on compact manifolds of negative sectional curvature. Moreover, for a manifold $M$ like that, one can find a positive constant $\eta$ such that either the norm of the second fundamental tensor of any foliation on $M$ or the norm of its covariant derivative has to exceed $\eta$ somewhere. We end with some questions and remarks $(\S 7)$. 
Finally, we would like to say that we began this work during a stay at the University of Sao Paulo where Question B of $\S 7$ was posed and discussed with Fabiano Brito, Remi Langevin and Waldyr Oliva. Also, Problem D of $\S 7$ had already been discussed with Etienne Ghys and Remi Langevin.

\section{Preliminaries}

Throughout the paper, $M$ is an oriented $C^{\infty}$-manifold with a $C^{3}$-Riemannian structure $g=\langle\cdot, \cdot\rangle . F$ is an oriented $C^{3}$-foliation of $M, p=\operatorname{dim} F, n=\operatorname{dim} M$, $0<p<n$. For any $x$ in $M, L_{x}$ denotes the leaf of $F$ which passes through $x . T F$ and $S F$ denote the tangent bundle and the unit tangent bundle of $F$, respectively.

We always assume that the leaves of $F$ are complete with respect to the Riemannian structure induced from $M$. For any $v$ of $T_{x} F, c_{v}: R \rightarrow L_{x}$ is the maximal geodesic satisfying $c_{v}(0)=x$ and $\dot{c}_{v}(0)=v$. The maps $\varphi_{t}, t \in R$, of $T F$ into itself are defined by $\varphi_{i}(v)=c_{v}(t)$. They form a flow $\varphi=\left(\varphi_{i}\right)$ which is called the geodesic flow of $F$. Since $\left|\varphi_{t} v\right|=|v|, \varphi$ preserves the bundle $S F$ and induces the geodesic flow on $S F$ which is also denoted by $\varphi$.

The Levi-Civita connection on $M$, its curvature tensor and the sectional curvature of $M$ are denoted here by $\nabla, R$ and $K$, respectively. $\nabla$ induces a connection $\nabla^{F}$ in the bundle $T F$. We have

$$
\nabla_{X}^{F} Y=\left(\nabla_{X} Y\right)^{\mathrm{T}}
$$

where $X$ is a vector field on $M, Y$ is a section of $T F$ and

$$
v=v^{\mathbf{T}}+v^{\perp}
$$

is the decomposition of a vector $v \in T M$ into the components tangential to and orthogonal to $F$.

The second fundamental form of $F$ can be considered either as a section $A$ of the bundle Hom $\left(T^{\perp} F \otimes T F, T F\right)$ or as a section $B$ of Hom $(T F \otimes T F, T M)$, where $T^{\perp} F$ is the orthogonal complement of $T F$ in $T M$. We have

and

$$
A(N, X)=-\left(\nabla_{X} N\right)^{\mathrm{T}}
$$

$$
B(X, Y)=\left(\nabla_{X} Y\right)^{\perp} .
$$

for a section $N$ of $T^{\perp} F$ and sections $X$ and $Y$ of $T F$. Similarly, the second fundamental tensor $B^{\perp}$ of $T^{\perp} F$ is given by

$$
B^{\perp}(X, Y)=\frac{1}{2}\left(\nabla_{X} Y+\nabla_{Y} X\right)^{T}
$$

for sections $X$ and $Y$ of $T^{\perp} F$.

The connections $\nabla$ and $\nabla^{F}$ induce a connection $\tilde{\nabla}$ in the bundle $\operatorname{Hom}(T F \otimes$ $T F, T M)$. We have

$$
\begin{aligned}
\left(\tilde{\nabla}_{Z} B\right)(X, Y)= & \nabla_{Z} B(X, Y) \\
& -B\left(\nabla_{Z}^{F} X, Y\right)-B\left(X, \nabla_{Z}^{F} Y\right) .
\end{aligned}
$$

Note that $\tilde{\nabla}$ differs from the connection $\bar{\nabla}$ which appears in the Codazzi equations $[\mathbf{K N}] . \bar{\nabla}$ is the connection in the bundle Hom $\left(T F \otimes T F, T^{\perp} F\right)$ induced by $\nabla^{F}$ and 
$\nabla^{\perp}$, the natural connection in $T^{\perp} F$. We have

$$
\left(\tilde{\nabla}_{Z} B\right)(X, Y)=\left(\bar{\nabla}_{Z} B\right)(X, Y)+\left(\nabla_{Z} B(X, Y)\right)^{\top} \text {. }
$$

Now, given $v$ in $T_{x} F$ we can consider the linear transformation

$$
T_{x} F \ni w \mapsto B(v, w) .
$$

Its norm is denoted here by $|B(v)|$. We have

$$
|B(v)|=\sup \{|B(v, w)| ;|w|=1\} .
$$

Similarly, $|\tilde{\nabla} B(v, w)|\left(v, w \in T_{x} F\right)$ denotes the norm of the linear map

$$
T_{x} F \ni u \mapsto\left(\tilde{\nabla}_{u} B\right)(v, w) .
$$

Also, if $v \in S_{x} F$, then we denote by $K(v)$ (respectively, by $|K(v)|$ ) the maximum of sectional curvatures of $M$ (respectively, maximum of their absolute values) over the set of all planes $\sigma \subset T_{x} M$ containing $v$.

Finally, we equip $T F$ (and $S F$ as well) with two Riemannian metrics which arise in a natural way. The first one, $\tilde{g}=\langle\cdot, \cdot\rangle^{\sim}$, is defined as the metric induced from $T M$. Recall that $T M$ carries the metric (denoted here also by $\tilde{\mathrm{g}}$ ) given by

$$
\tilde{\boldsymbol{g}}(\xi, \eta)=\left\langle\pi_{*} \xi, \pi_{*} \eta\right\rangle+\langle C \xi, C \eta\rangle
$$

where $\xi, \eta \in T_{v} T M, \pi: T M \rightarrow M$ is the canonical projection and $C: T T M \rightarrow T M$ is the connection map of $\nabla$. Recall also (see [GKM]) that $C$ maps linearly $T_{v} T M$ onto $T_{x} M(x=\pi(v))$, coincides with the canonical identification $T_{v}\left(T_{x} M\right) \approx T_{x} M$ on the space of vertical vectors and satisfies

$$
C\left(X_{*} u\right)=\nabla_{u} X
$$

for any $u$ of $T M$ and any vector field $X$ on $M$. On the other hand, we can define the connection map $C^{F}$ of $\nabla^{F}$. $C^{F}$ maps $T_{v} T F$ linearly onto $T_{x} F$ where $v \in T_{x} F$ coincides with the canonical identification $T_{v}\left(T_{x} F\right) \approx T_{x} F$ on the space of vertical vectors and satisfies

$$
C^{F}\left(X_{*} u\right)=\nabla_{u}^{F} X
$$

for any $u$ of $T M$ and any section $X$ of $T F$. With this map we may put

$$
\tilde{g}_{F}(\xi, \eta)=\left\langle\pi_{*} \xi, \pi_{*} \eta\right\rangle+\left\langle C^{F} \xi, C^{F} \eta\right\rangle
$$

for all $\xi$ and $\eta$ of $T_{v} T F\left(v \in T F\right.$ ). $g_{F}=\langle\cdot, \cdot\rangle_{F}$ is also a Riemannian metric on TF. The metrics $\tilde{g}$ and $\tilde{g}_{F}$ coincide on $T F$ when the foliation $F$ is totally geodesic (i.e., $B=0$ ). If $M$ is compact, the metrics induced by $\tilde{g}$ and $\tilde{g}_{F}$ on $S F$ are quasi-isometric, so from the dynamical point of view they are equivalent. In this paper, we shall sometimes use one of them, sometimes the other.

Let us note that the vector field $V$ generated on $T F$ by the flow $\varphi$ has the following properties:

(i) $\pi_{*} \circ V=$ id $_{T F}$,

(ii) $C^{F} \circ V=0$,

(iii) $V \circ \mu_{r}=r \cdot \mu_{r *} \circ V, r \in \mathbb{R}$, where $\mu_{r}: T F \rightarrow T F$ is multiplication by $r$.

These properties of $V$ follow immediately from the analogous properties (see [GKM]) of geodesic flows of Riemannian manifolds because our flow $\varphi$ restricted to the tangent bundle $T L$ of a leaf $L$ coincides with the geodesic flow of $L$. 


\section{Jacobi fields}

Let us consider a curve $v:(-\varepsilon, \varepsilon) \rightarrow T F$ and the mapping $f: \mathbb{R} \times(-\varepsilon, \varepsilon) \rightarrow M$ given by $f(t, s)=\exp ^{F} t v(s)$, where $\exp ^{F}$ is the exponential map of $\nabla^{F}: \exp ^{F}(u)=c_{u}(1)$ for any $u$ of $T F$. Put

$$
X=f_{*}(d / d t), \quad Y=f_{*}(d / d s)
$$

and $Z(t)=Y(t, 0)$ for $t \in \mathbb{R} . Z$ is a vector field along the geodesic $c=c_{v(0)}$.

Since the vector fields $d / d t$ and $d / d s$ commute, and the torsion of $\nabla$ vanishes, we have

and

$$
R(Y, X) X=\nabla_{d / d s} \nabla_{d / d t} X-\nabla_{d / d t} \nabla_{d / d s} X
$$

$$
\nabla_{d / d s} X=\nabla_{d / d t} Y
$$

Also,

$$
\nabla_{d / d t} X=\nabla_{d / d t}^{F} X+B(X, X)=B(X, X)
$$

because the trajectories of $X$ are geodesics on leaves of $F$. These equalities together with (1) yield the equality

$$
R(Y, X) X=\left(\tilde{\nabla}_{d / d s} B\right)(X, X)+2 B\left(\nabla_{d / d t}^{F} Y, X\right)-\nabla_{d / d t} \nabla_{d / d t} Y,
$$

which assumes the shape of

$$
Z^{\prime \prime}-2 B\left(Z^{\prime \mathrm{T}}, \dot{c}\right)-\left(\tilde{\nabla}_{Z} B\right)(\dot{c}, \dot{c})-R(\dot{c}, Z) \dot{c}=0
$$

along $c$. Here $Z^{\prime}=\nabla_{d / d t} Z$ and $Z^{\prime \prime}=\left(Z^{\prime}\right)^{\prime}$.

Now, put $\xi=\dot{v}(0)$. Then

and

$$
Z(0)=(\pi \circ v)(0)=\pi_{*} \xi
$$

$$
\begin{aligned}
Z^{\prime}(0) & =\nabla_{d / d t} Y(0,0)=\nabla_{d / d s} X(0,0) \\
& =\nabla_{d / d s} v(0)=C\left(v_{*}(d / d s)\right)=C \xi .
\end{aligned}
$$

From the theory of ordinary differential equations, it follows that for any $\xi$ of TTM there exists a unique solution $Z_{\xi}$ of (3) satisfying $Z_{\xi}(0)=\pi_{*} \xi$ and $Z_{\xi}^{\prime}(0)=C \xi$. However, only those with $\xi \in T T F$ correspond to variations of geodesic considered above. We call them Jacobi fields (for F) here. It is clear that Jacobi fields form a vector space of dimension $n+p$.

Remark 1. If $Z$ is a Jacobi field for $F$ tangential everywhere to $F$, then $Z$ is a Jacobi field on a leaf of $F$. This can be seen easily from the construction of the Jacobi fields described above. Also, using equality (2) and the Codazzi and Gauss equations one can see that if $W$ and $Z$ are vector fields along a geodesic $c$ and $W$ is tangential to $F$, then

$$
\begin{aligned}
\left\langle Z^{\prime \prime}-\right. & \left.2 B\left(Z^{\prime \top}, \dot{c}\right)-\left(\tilde{\nabla}_{Z} B\right)(\dot{c}, \dot{c})-R(\dot{c}, Z) \dot{c}, W\right\rangle \\
= & \left\langle\nabla_{\dot{c}}^{F} \nabla_{\dot{c}}^{F} Z-R^{F}\left(\dot{c}, z^{\mathrm{T}}\right) \dot{c}, W\right\rangle \\
& -2\left\langle\left(\bar{\nabla}_{\dot{c}} B\right)(W, \dot{c}), Z^{\perp}\right\rangle+\left\langle\left(\bar{\nabla}_{W} B\right)(\dot{c}, \dot{c}), Z^{\perp}\right\rangle \\
& +\left\langle B(\dot{c}, \dot{c}), \nabla_{Z^{\perp}} W\right\rangle-2\left\langle B(\dot{c}, W), \nabla_{\dot{c}}^{\perp} Z^{\perp}\right\rangle
\end{aligned}
$$


This equality shows that: (1) if $Z^{\perp}=0$ and $Z$ is a Jacobi field for $F$, then it is a Jacobi field on a leaf, $(2)$ if $F$ is totally geodesic, then the tangent component $Z^{\top}$ of any Jacobi field for $F$ is a Jacobi field on a leaf.

LEMMA 1. If $Z$ is a Jacobi field (for $F$ ) along a geodesic $c$, then $\left\langle Z^{\prime}, \dot{c}\right\rangle=$ constant.

Proof. With the notation used in the beginning of this section we have

$$
\begin{aligned}
& \left\langle\left(\tilde{\nabla}_{Y} B\right)(X, X), X\right\rangle+\left\langle\nabla_{d / d t} Y, B(X, X)\right\rangle \\
& =\left\langle\nabla_{d / d s} B(X, X), X\right\rangle-2\left\langle B\left(\nabla_{d / d s}^{F} X, X\right), X\right\rangle+\left\langle\nabla_{d / d t} Y, B(X, X)\right\rangle \\
& =\left\langle B(X, X), \nabla_{d / d t} Y-\nabla_{d / d s} X\right\rangle=0 .
\end{aligned}
$$

Therefore,

$$
\begin{aligned}
\frac{d}{d t}\left\langle Z^{\prime}, \dot{c}\right\rangle & =\left\langle Z^{\prime \prime}, \dot{c}\right\rangle+\left\langle Z^{\prime}, B(\dot{c}, \dot{c})\right\rangle \\
& =\left\langle\left(\tilde{\nabla}_{Z} B\right)(\dot{c}, \dot{c}), \dot{c}\right\rangle+\left\langle Z^{\prime}, B(\dot{c}, \dot{c})\right\rangle=0 .
\end{aligned}
$$

Remark 2. Since $(d / d t)\langle Z, \dot{c}\rangle=\left\langle Z^{\prime}, \dot{c}\right\rangle+\langle B(\dot{c}, \dot{c}), Z\rangle$ we cannot claim (as for Jacobi fields on Riemannian manifolds) that $Z$ is constantly orthogonal to $c$ if $Z(0)$ and $Z^{\prime}(0)$ are. This makes our calculation slightly different from that for the geodesic flow of a Riemannian manifold.

LEMMA 2. For any $\xi$ of TTF and any $t$ of $\mathbb{R}$ we have

$$
\pi_{*} \varphi_{t *} \xi=Z_{\xi}(t) \text { and } C \varphi_{t *} \xi=Z_{\xi}^{\prime}(t)
$$

Proof. If $\xi=\dot{v}(0)$ for a curve $v:(-\varepsilon, \varepsilon) \rightarrow T F$, then

and

$$
\begin{aligned}
\pi_{*} \varphi_{t *} \xi & =\left.(d / d s)\left(\pi \circ \varphi_{t} \circ v\right)\right|_{s=0} \\
& =\left.(d / d s)\left(\exp ^{F} t v(s)\right)\right|_{s=0}=Z_{\xi}(t)
\end{aligned}
$$

$$
\begin{aligned}
C \varphi_{t *} \xi & =C\left(\left(s \mapsto \varphi_{t}(v(s))\right)^{\bullet}(0)\right) \\
& =C\left((s \mapsto X(t, s))^{\bullet}(0)\right)=\left(\nabla_{d / d s} X\right)(t, 0) \\
& =\left(\nabla_{d / d t} Y\right)(t, 0)=Z_{\xi}^{\prime}(t) .
\end{aligned}
$$

CoRollary 1. $\left\langle\varphi_{i *} \xi, \varphi_{t *} \eta\right\rangle^{\sim}=\left\langle Z_{\xi}(t), Z_{\eta}(t)\right\rangle+\left\langle Z_{\xi}^{\prime}(t), Z_{\eta}^{\prime}(t)\right\rangle$ for any $\xi, \eta \in T_{v} T F$ and $v \in T F$.

Lemma 2 and Corollary 1 show that to study the dynamical properties of the flow $\varphi$ we should investigate the behaviour of Jacobi fields.

\section{An invariant measure}

The existence of invariant measures for a dynamical system is an old and still important question. For example, in [PI] and [GLW] one can find conditions sufficient for a pseudo-group of local diffeomorphisms to admita non-trivial invariant measure. In the case of a flow on a manifold, a reasonable question is to look for a smooth (i.e. absolutely continuous with respect to the Riemannian volume) invariant measure. The geodesic flow on a Riemannian manifold $(M, g)$ always 
preserves the Riemannian volume of $(T M, \tilde{g})$. Zeghib $[Z]$ proved that the geodesic flow of a totally geodesic foliation of a compact locally symmetric space always admits an invariant smooth measure. In this context we prove here the following.

THEOREM 1. The geodesic flow $\varphi$ of a foliation $F$ preserves the Riemannian volume of $\left(S F, \tilde{g}_{F}\right)$ if and only if $F$ is transversely minimal, i.e. trace $B^{\perp}=0$.

Proof. Denote by $\Omega$ and $\omega$, respectively, the volume forms on $T F$ and $S F$ with the Riemannian metric $\tilde{g}_{F}$. On $S F$ we have

$$
\Omega=\omega \wedge \theta
$$

where $\theta$ is the one-form on $S F$ given by $\theta(\xi)=\langle W, \xi\rangle \tilde{g}_{F}$ with $W$ being the vertical field on $T F$ given by

$$
W(u)=(t \mapsto t u)^{\cdot}(1) \quad(u \in T F) .
$$

Since the vector field $W$ corresponds to the flow $\left(\mu_{e^{t}}\right)$ and

$$
L_{V} \Omega=L_{V} \omega \wedge \theta+\omega \wedge L_{V} \theta,
$$

so

$$
[V, W]=\lim _{t \rightarrow 0} \frac{1}{t}\left(V-\mu_{\mathrm{e}^{i}} * V \mu_{\mathrm{e}^{-r}}\right)=V,
$$

$L_{V} \theta=0$ and consequently

$$
L_{V} \Omega=0 \quad \text { iff } \quad L_{V} \omega=0 .
$$

Now, take a local orthonormal frame $X_{1}, \ldots, X_{n}$ of vector fields on $M$ such that $X_{i}$ is tangential to $F$ for $i=1, \ldots, p$. Denote by $E_{i}(i=1, \ldots, p)$ the vertical lift of $X_{i}$ and by $E_{p+j}(j=1, \ldots, n)$ the horizontal lift of $X_{j}$. Then

and

$$
\pi_{*} E_{i}=0 \quad \text { and } \quad C_{F} E_{i}=X_{i} \quad(i=1, \ldots, p)
$$

$$
\pi_{*} E_{p+j}=X_{j} \quad \text { and } \quad C_{F} E_{p+j}=0 \quad(j=1, \ldots, n) .
$$

The fields $E_{1}, \ldots, E_{n+p}$ form a local orthonormal frame on $T F$, so

$$
\Omega\left(E_{1}, \ldots, E_{n+p}\right)=1
$$

and

$$
\begin{aligned}
L_{V} \Omega\left(E_{1}, \ldots, E_{n+p}\right)= & -\sum_{i=1}^{2 p} \Omega\left(E_{1}, \ldots,\left[V, E_{i}\right], \ldots, E_{n+p}\right) \\
& -\sum_{j=2 p+1}^{n+p} \Omega\left(E_{1}, \ldots,\left[V, E_{j}\right], \ldots, E_{n+p}\right) .
\end{aligned}
$$

The first sum in (5) vanishes because it is equal to

$$
L_{V \mid L} \Omega_{L}\left(E_{1}, \ldots, E_{2 p}\right),
$$

where $\Omega_{L}$ is the Riemannian volume form on the tangent bundle $T L$ of a leaf $L$, and the geodesic flow on $T L$ preserves $\Omega_{L}$. The second sum in (5) is equal to

$$
\sum_{j=p+1}^{n}\left\langle\pi_{*}\left[V, E_{n+j}\right], X_{j}\right\rangle
$$

To calculate it we need the following. 
LEMMA 3. Let $X$ be a vector field on $M$ and $X^{h}$ its horizontal lift to TF. Then for any $v$ of $T_{x} F$ we have

$$
\pi_{*}\left[V, X^{\mathrm{h}}\right](v)=[Z, X](x),
$$

where $Z$ is any vector field on $M$ satisfying $Z\left(\psi_{t} x\right)=\tilde{\psi}_{t} v(-\varepsilon<t<\varepsilon)$ for the (local) flows $\left(\psi_{t}\right)$ of $X$ and $\left(\tilde{\psi}_{\imath}\right)$ of $X^{\mathrm{h}}$. Consequently,

$$
\left\langle\pi_{*}\left[V, X^{\mathrm{h}}\right](v), X(x)\right\rangle=\left\langle B^{\perp}(X, X)(x), v\right\rangle
$$

when $X$ is orthogonal to $F$ and $|X|=$ constant.

Proof. Since $\pi_{*} \circ X^{\mathrm{h}}=X \circ \pi, \pi \circ \tilde{\psi}_{\mathrm{t}}=\psi_{\mathrm{t}} \circ \pi$ for any $t$. Therefore,

$$
\begin{aligned}
\pi_{*}\left(\left[V, X^{\mathrm{h}}\right](v)\right) & =\lim _{t \rightarrow 0} \frac{1}{t}\left(\pi_{*} V(v)-\pi_{*} \tilde{\psi}_{t^{*}} V\left(\tilde{\psi}_{-t} v\right)\right) \\
& =\lim _{t \rightarrow 0} \frac{1}{t}\left(v-\psi_{t^{*}} \pi_{*} V\left(\tilde{\psi}_{-t} v\right)\right)=\lim _{t \rightarrow 0} \frac{1}{t}\left(v-\psi_{t^{*}} \tilde{\psi}_{-t} v\right) \\
& =\lim _{t \rightarrow 0} \frac{1}{t}\left(Z(x)-\psi_{t^{*}} Z\left(\psi_{-t} x\right)\right)=[Z, X](x) .
\end{aligned}
$$

Consequently,

$$
\begin{aligned}
\left\langle\pi_{*}\left[V, X^{\mathrm{h}}\right](v), X(x)\right\rangle & =\langle[Z, X], X\rangle(x) \\
& =\left\langle\nabla_{Z} X, X\right\rangle(x)-\left\langle\nabla_{X} Z, X\right\rangle(x)=\left\langle Z, \nabla_{X} X\right\rangle(x)
\end{aligned}
$$

because $X$ is orthogonal to $F$ while $Z$ is tangential to $F$ along the trajectory of $X$ passing through $x$. Moreover,

$$
\left\langle Z, \nabla_{X} X\right\rangle(x)=\left\langle B^{\perp}(X, X)(x), v\right\rangle .
$$

In order to complete the proof of Theorem 1 we should just apply formula (7) to the sum (6).

Remark 3. The condition trace $B^{\perp}=0$ is equivalent to the following:

(*) $L_{X} \omega^{\perp}=0$ for any vector field $X$ tangential to $F$, where $\omega^{\perp}$ is the volume form of $T^{\perp} F$ induced by $g$, i.e. $\omega^{\perp}$ is a $q$-form on $M(q=\operatorname{codim} M)$ given by

$$
\omega^{\perp}\left(v_{1}, \ldots, v_{q}\right)=\operatorname{det}\left[\left(v_{i}, e_{j}\right\rangle ; i, j \leq q\right] \text {, }
$$

$e_{1}, \ldots, e_{q}$ being an orthonormal frame of $T^{\perp} F$. In other words, $\omega^{\perp}$ determines a holonomy invariant measure in the sense of [PI]. More precisely, if $T_{k}(k=1,2)$ is a submanifold transverse to $F$ and $h: T_{1} \rightarrow T_{2}$ is a holonomy map of $F$, then

$$
h^{*}\left(\omega^{\perp} \mid T_{2}\right)=\omega^{\perp} \mid T_{1} \text {. }
$$

Examples. Any Riemannian foliation is transversely geodesic $\left(B^{\perp}=0\right)$, so it is transversely minimal. In codimension one, the converse is true: every transversely minimal foliation of $(M, g)$ is Riemannian with $g$ being bundle-like. If the bundle $T^{\perp} F$ is integrable, then $F$ is transversely minimal iff the leaves of the orthogonal foliation are minimal. 


\section{Lyapunov exponents}

Let $\left(f_{t}\right)$ be an arbitrary flow on a Riemannian manifold $(M, g)$. If $x \in M$ and $0 \neq v \in T_{x} M$, then we put

$$
\lambda(v)=\limsup _{t \rightarrow+\infty} \frac{1}{t} \log \left|f_{t *} v\right| .
$$

Recall that $\lambda(v)$ is called the Lyapunov exponent of $\left(f_{t}\right)$ in the direction of $v$. From the definition it is clear that the function $\lambda: T_{x} M \backslash\{0\} \rightarrow \mathbb{R}$ admits at most $n=\operatorname{dim} M$ values $\lambda_{1}<\cdots<\lambda_{k}$ and that there is a filtration $L_{1}(x) \subset L_{2}(x) \subset \cdots \subset L_{k}(x)=T_{x} M$ such that each of $L_{j}(x)$ is a linear subspace of $T_{x} M$ and $\lambda(v)=\lambda_{j}$ iff $v \in L_{j}(x) \backslash L_{j-1}(x)$ $\left(L_{0}(x)=0\right)$. Clearly, $\lambda(v)=0$ when $v$ is tangential to the flow.

If $M$ is compact, we have Oseledets' Multiplicative Ergodic Theorem ([O], see also $[\mathbf{M}]$ and [Wa]) which says that $M$ contains a Borel set $\Lambda$ such that (i) $\mu(\Lambda)=1$ for any Borel $f_{i}$-invariant probability measure on $M$ and (ii) for any $x$ in $\Lambda$ there are unique constants $\lambda_{1}(x)<\cdots<\lambda_{k}(x)$ and unique decomposition $T_{x} M=E_{1}(x) \oplus$ $\cdots \oplus E_{k}(x)$ for which

$$
\lim _{t \rightarrow \pm \infty} \frac{1}{t} \log \left|f_{t^{*}} v\right|=\lambda_{j}(x)
$$

if and only if $v \in E_{j}(x)$.

In [M] elements of the set $\Lambda$ are called regular for the flow $\left(f_{t}\right)$.

In this section, we will estimate Lyapunov exponents of the geodesic flow of $F$. For this purpose we shall apply Corollary 1 of $\S 2$. Our calculation is analogous to that of [S] with one difference: we have to remember that Jacobi fields orthogonal to a geodesic at one point do not remain orthogonal to it all the time.

First, let us put

$$
\Phi(u)=4|B(u)|+|\tilde{\nabla} B(u, u)|+|K(u)|
$$

for $u \in T F$, take $\xi \in T_{v} T F(\xi \neq 0)$ and put

$$
z_{a}(t)=a|Z(t)|^{2}+\frac{1}{a}\left|Z^{\prime}(t)\right|^{2} \quad(t \in \mathbb{R})
$$

where $a$ is a positive constant and $Z=Z_{\xi}$ is a Jacobi field along the geodesic $c=c_{v}$. We have

$$
\begin{aligned}
z_{a}^{\prime}= & 2 a\left\langle Z, Z^{\prime}\right\rangle+\frac{2}{a}\left\langle Z^{\prime}, Z^{\prime \prime}\right\rangle=2 a\left\langle Z, Z^{\prime}\right\rangle \\
& +\frac{4}{a}\left\langle B\left(Z^{\prime \mathbf{T}}, \dot{c}\right), Z^{\prime}\right\rangle+\frac{2}{a}\left\langle\left(\tilde{\nabla}_{Z} B\right)(\dot{c}, \dot{c}), Z^{\prime}\right\rangle \\
& +\frac{2}{a}\left\langle R(\dot{c}, Z) \dot{c}, Z^{\prime}\right\rangle \leq 2 a|Z| \cdot\left|Z^{\prime}\right| \\
& +\frac{4}{a}|B(\dot{c})| \cdot\left|Z^{\prime}\right|^{2}+\frac{2}{a}|\tilde{\nabla} B(\dot{c}, \dot{c})| \cdot|Z| \cdot\left|Z^{\prime}\right| \\
& +\frac{2}{a}|K(\dot{c})| \cdot|Z| \cdot\left|Z^{\prime}\right| \leq\left(a+\frac{1}{a} \Phi(\dot{c})\right) \cdot z_{a} .
\end{aligned}
$$


Therefore,

$$
\left(\log z_{a}\right)^{\prime} \leq a+\frac{1}{a} \Phi(\dot{c})
$$

and

$$
\limsup _{t \rightarrow+\infty} \frac{1}{t} \log z_{a}(t) \leq a+\frac{1}{a} \limsup _{t \rightarrow+\infty} \frac{1}{t} \int_{0}^{t} \Phi(\dot{c}(s)) d s .
$$

Now, if $f(t)=|Z(t)|^{2}+\left|Z^{\prime}(t)\right|^{2}$, then $f \leq \alpha \cdot z_{a}$ for some positive constant $\alpha$. Therefore,

$$
\limsup _{t \rightarrow+\infty} \frac{1}{t} \log f(t) \leq a+\frac{1}{t} \limsup _{t \rightarrow+\infty} \frac{1}{t} \int_{0}^{t} \Phi(\dot{c}(s)) d s
$$

for any $a>0$. Estimation (10) is the best when

$$
a=\left[\limsup _{t \rightarrow+\infty} \frac{1}{t} \int_{0}^{t} \Phi(\dot{c}(s)) d s\right]^{1 / 2} .
$$

With this constant we get

$$
\limsup _{t \rightarrow+\infty} \frac{1}{t} \log f(t) \leq 2\left[\limsup _{t \rightarrow+\infty} \frac{1}{t} \int_{0}^{t} \Phi(\dot{c}(s)) d s\right]^{1 / 2} .
$$

Next, let us assume that $\pi_{*} \xi \perp \dot{c}(0)$ and consider the function $y_{a}: \mathbb{R} \rightarrow \mathbb{R}$ given by

$$
y_{a}(t)=a|Z(t)|^{2}-\langle Z(t), \dot{c}(t)\rangle^{2},
$$

where $a \in(0 ; 1)$ is fixed. Assume also that $\left\langle Z^{\prime}(0), \dot{c}(0)\right\rangle=0$, i.e. that (Lemma 1) $\left\langle Z^{\prime}, \dot{c}\right\rangle \equiv 0$. Then

$$
y_{a}^{\prime}=2 a\left\langle Z, Z^{\prime}\right\rangle-2\langle Z, \dot{c}\rangle\langle Z, B(\dot{c}, \dot{c})\rangle
$$

and

$$
\begin{aligned}
\frac{1}{2} y_{a}^{\prime \prime}= & a\left\langle Z, Z^{\prime \prime}\right\rangle+a\left|Z^{\prime}\right|^{2}-\langle Z, B(\dot{c}, \dot{c})\rangle^{2} \\
& -\langle Z, \dot{c}\rangle\left\langle Z^{\prime}, B(\dot{c}, \dot{c})\right\rangle-\langle Z, \dot{c}\rangle\left\langle Z,\left(\tilde{\nabla}_{\dot{c}} B\right)(\dot{c}, \dot{c})\right\rangle
\end{aligned}
$$

From (3) we get

$$
\begin{aligned}
\frac{1}{2} y_{a}^{\prime \prime} \geq & -a K(\dot{c}) y_{a}+\left[| Z | ^ { 2 } \left(\left(a^{2}-a\right) K(\dot{c})\right.\right. \\
& \left.-(a+1)|\tilde{\nabla} B(\dot{c}, \dot{c})|-|B(\dot{c})|^{2}\right) \\
& \left.+|Z| \cdot\left|Z^{\prime}\right|(2 a-1) \cdot|B(\dot{c})|+\left|Z^{\prime}\right|^{2} a\right] .
\end{aligned}
$$

Assume now that the sectional curvature of $M$ is negative, $K_{M} \leq K_{0}<0$. Then for any $\delta>0$ there exists $\eta>0$ such that the sum in the square brackets in (12) is always non-negative for any $a \in(\delta, 1-\delta)$ if only $|B(\dot{c})|<\eta$ and $|\tilde{\nabla} B(\dot{c}, \dot{c})|<\eta$. For these $a$ we have

$$
y_{a}^{\prime \prime} \geq-2 a K(\dot{c}) y_{a} \geq-2 a K_{0} y_{a} .
$$

If, moreover, $\xi$ is horizontal $(C \xi=0)$, then $y_{a}(0)=a\left|\pi_{*} \xi\right|^{2}>0$ and $y_{a}^{\prime}(0)=0$. So, it is easy to see that in this case

$$
y_{a}(t) \rightarrow+\infty \text { when } t \rightarrow+\infty \text {. }
$$


Now, put

$$
\Psi_{a}(u)=(1-|B(u)|)(K(u)(a-1)-|B(u)|-|\tilde{\nabla} B(u, u)|) \quad(u \in T F)
$$

and

$$
g(t)=\left\langle Z(t), Z^{\prime}(t)\right\rangle \quad(t \in \mathbb{R})
$$

Then

$$
\begin{aligned}
g^{\prime}= & \left\langle Z, Z^{\prime \prime}\right\rangle+\left|Z^{\prime}\right|^{2} \geq-K(\dot{c}) y_{a} \\
& +\left[|Z|^{2}(K(\dot{c})(a-1)-|\tilde{\nabla} B(\dot{c}, \dot{c})|)\right. \\
& \left.-2|Z| \cdot\left|Z^{\prime}\right| \cdot|B(\dot{c})|+\left|Z^{\prime}\right|^{2}\right]
\end{aligned}
$$

and if $\xi$ and $a$ are such that condition (13) holds, then for $t$ big enough we have

$$
\begin{aligned}
g^{\prime}(t) \geq & |Z(t)|^{2}(K(\dot{c}(t))(a-1)-|\tilde{\nabla} B(\dot{c}(t), \dot{c}(t))| \\
& -|B(\dot{c}(t))|)+\left|Z^{\prime}(t)\right|^{2}(1-|B(\dot{c}(t))|) \\
\geq & 2 g(t) \sqrt{\Psi_{a}(\dot{c}(t))}
\end{aligned}
$$

if $|B(\dot{c})| \leq 1$ and $|B(\dot{c})|+|\tilde{\nabla} B(\dot{c}, \dot{c})| \leq K(\dot{c})(a-1)$. In this case, $g(t)>0$ for $t$ big enough and

$$
\limsup _{t \rightarrow+\infty} \frac{1}{t} \log g(t) \geq 2 \limsup _{t \rightarrow+\infty} \frac{1}{t} \int_{0}^{t}\left(\Psi_{a}(\dot{c}(s))\right)^{1 / 2} d s .
$$

Finally, since $f(t) \geq 2 g(t)$,

$$
\limsup _{t \rightarrow+\infty} \frac{1}{t} \log f(t) \geq 2 \limsup _{t \rightarrow+\infty} \frac{1}{t} \int_{0}^{t}\left(\Psi_{a}(\dot{c}(s))\right)^{1 / 2} d s .
$$

Let us recapitulate the above results as follows.

THEOREM 2. The Lyapunov exponents $\lambda(\xi)$ of the flow $\varphi$ satisfy

$$
\lambda(\xi) \leq\left[\limsup _{t \rightarrow+\infty} \frac{1}{t} \int_{0}^{t} \Phi\left(\varphi_{s} v\right) d s\right]^{1 / 2},
$$

where $\xi \in T_{v} S F$ and $\Phi$ is given by (9). Moreover, if the sectional curvature of $M$ is negative, if $\left|B\left(\varphi_{s} v\right)\right|$ and $\left|\tilde{\nabla} B\left(\varphi_{s} v, \varphi_{s} v\right)\right|$ are small enough for any s, and if $\xi$ is horizontal, then there exists $a \in(0 ; 1)$ such that

$$
\lambda(\xi) \geq \limsup _{t \rightarrow+\infty} \frac{1}{t} \int_{0}^{t}\left(\Psi_{a}\left(\varphi_{s} v\right)\right)^{1 / 2} d s
$$

with $\Psi_{a}$ given by (14).

Proof. From Corollary 1 and (8), it follows immediately that (16) is equivalent to (11) while (17) is to (15).

\section{Entropy estimate}

In this section, $M$ is assumed to be compact. Denote by $\Lambda$ the set of all points regular for $\varphi$. For any $v$ of $\Lambda$ denote by $\chi(v)$ the sum of all positive Lyapunov exponents of $\varphi$ counted with their multiplicities:

$$
\chi(v)=\sum_{\left\{i ; \lambda_{i}(v)>0\right\}} \lambda_{i}(v) \cdot \operatorname{dim} E_{i}(v)
$$


with notation of $\S 4$. The measure entropy $h_{\mu}(\varphi)$ of $\varphi$ with respect to any $\varphi$-invariant Borel probability measure $\mu$ satisfies (see [R])

$$
h_{\mu}(\varphi) \geq \int_{S F} \chi d \mu .
$$

THEOREM 3. The measure entropy of $\varphi$ with respect to any invariant measure $\mu$ satisfies

$$
h_{\mu}(\varphi) \leq \frac{1}{2}(n+p-2)\left[\int_{S F} \Phi d \mu\right]^{1 / 2} \text {. }
$$

Therefore, the topological entropy of $\varphi$ satisfies

$$
h_{\text {top }}(\varphi) \leq \frac{1}{2}(n+p-2) \max _{S F} \Phi^{1 / 2} .
$$

Proof. First, for any point $v$ regular for $\varphi$ put

and

$$
\begin{aligned}
& E^{u}(v)=\underset{\left\{i ; \lambda_{i}(v)>0\right\}}{\bigoplus} E_{i}(v), \\
& E^{s}(v)=\underset{\left\{i ; \lambda_{i}(v)<0\right\}}{\bigoplus} E_{i}(v)
\end{aligned}
$$

$$
E^{0}(v)=\left\{\xi ; \lim _{t \rightarrow \pm \infty} \frac{1}{t} \log \left|\varphi_{t^{*}} \xi\right|=0\right\} .
$$

Denote by $\sigma$ the isometry of $S F$ given by $\sigma(v)=-v$. Then

$$
\varphi_{-1} \circ \sigma=\sigma \circ \varphi_{t} \quad(t \in \mathbb{R})
$$

and therefore

$$
\sigma_{*} E^{u}(v)=E^{s}(\sigma(v)), \sigma_{*} E^{s}(v)=E^{u}(\sigma(v)) \quad \text { and } \quad \sigma_{*} E^{0}(v)=E^{0}(\sigma(v)) .
$$

Next, observe that the Birkhoff Ergodic Theorem asserts that the limit

$$
\alpha(v)=\lim _{t \rightarrow+\infty} \frac{1}{t} \int_{0}^{t} \Phi\left(\varphi_{s} v\right) d s
$$

exists almost everywhere and if $\mu$ is an ergodic invariant measure, then

$$
\alpha(v)=\int_{S F} \Phi d \mu
$$

for $\mu$-almost all $v$.

From (16), we get

$$
\chi(v) \leq \operatorname{dim} E^{u}(v) \cdot \alpha(v)^{1 / 2}
$$

and therefore

$$
\chi(v)+\chi(\sigma(v)) \leq\left[\operatorname{dim} E^{u}(v)+\operatorname{dim} E^{u}(\sigma(v))\right] \cdot \alpha(v)^{1 / 2}
$$

almost everywhere. Since $\operatorname{dim} E^{0}(v) \geq 1$ and $\operatorname{dim} E^{u}(\sigma(v))=\operatorname{dim} E^{s}(v)$ according to $(20)$, we have

$$
\chi(v)+\chi(\sigma(v)) \leq(n+p-2) \alpha(v)^{1 / 2}
$$

Also,

$$
\lim _{t \rightarrow \infty} \frac{1}{t} \log \left|\varphi_{-t^{*}} \sigma_{*} \xi\right|=\lim _{t \rightarrow \infty} \frac{1}{t}\left|\varphi_{t^{*}} \xi\right|
$$


so Lyapunov exponents of the flow $\varphi^{-1}=\left(\varphi_{-t}\right)$ at $\sigma(v)$ coincide with those of $\varphi$ at $v$. Therefore

Finally,

$$
h_{\mu}\left(\varphi^{-1}\right) \leq \int_{S F} \chi \circ \sigma d \mu .
$$

$$
\begin{aligned}
2 h_{\mu}(\varphi) & =h_{\mu}(\varphi)+h_{\mu}\left(\varphi^{-1}\right) \\
& \leq \int_{S F}(\chi+\chi \circ \sigma) d \mu \leq(n+p-2) \int_{S F} \alpha^{1 / 2} d \mu \\
& \leqslant(n+p-2)\left[\int_{S F} \alpha d \mu\right]^{1 / 2}=(n+p-2)\left[\int_{S F} \Phi d \mu\right]^{1 / 2}
\end{aligned}
$$

proving (18) for ergodic measures. Inequality (18) for an arbitrary measure follows now directly from the ergodic decomposition and Jacobs theorem (see, for example, [Wa]). Inequality (19) follows immediately from (18) because

$$
h_{\text {top }}(\varphi)=\sup _{\mu} h_{\mu}(\varphi) \text {. }
$$

Remark 3. If $F$ is a trivial foliation with the single leaf $M$, then $p=n$ and (18) reduces to

$$
h_{\mu}(\varphi) \leq(n-1)\left[\int_{S F}|K(u)| d \mu(u)\right]^{1 / 2},
$$

the inequality proved in $[S]$ in the case $K<0$.

6. Foliations of hyperbolic manifolds

In this section, we get the following application of estimates obtained in $\S 4$.

THEOREM 4. Let $M$ be a compact manifold of negative sectional curvature. Then there exists a positive number $\eta$ such that there are no non-trivial foliations of $M$ with the second fundamental form $B$ satisfying $|B|<\eta$ and $|\tilde{\nabla} B|<\eta$ everywhere on $M$. In particular, there are no totally geodesic foliations of $M$.

Proof. Assume that $\eta$ is such that the Lyapunov exponents $\lambda(\xi)$ of the geodesic flow $\varphi$ in the direction of horizontal vectors satisfy (17) for some $a>0$ if $|B|<\eta$ and $|\tilde{\nabla} B|<\eta$. In this case, $\lambda(\xi)>0$ for any horizontal vector $\xi$ of $T_{v} S F$ such that $\pi_{*} \xi \perp v$ and therefore

$$
\operatorname{dim} E^{u}(v) \geq n-1
$$

for any regular point $v$. From (20), it also follows that

$$
\operatorname{dim} E^{s}(v) \geq n-1
$$

and since $\operatorname{dim} E^{0}(v) \geq 1$ we get

$$
n+p-1=\operatorname{dim} T_{v} S F \geq 2 n-1
$$

and $n=p$.

Let us note that this argument is possible because - according to the Multiplicative Ergodic Theorem - the set of regular points is non-empty. If $M$ is non-compact, this is no longer true, so there exist many totally geodesic foliations of compete noncompact hyperbolic spaces. 


\section{Final remarks}

(A) Our entropy estimates are obtained in the way analogous to that of Sarnak [S] who also gives estimates from below. In the same way, one would use inequality (17) and the Pesin formula ([P], see also $[M])$ to estimate $h_{\mu}(\varphi)$ under some conditions on $M$ and $F$. However, one should be careful since, as we could see in the proof of Theorem 4 , we arrived at the conclusion $\operatorname{dim} F=\operatorname{dim} M$ assuming inequality (17) for all horizontal vectors. Also, the entropy estimates from [S] have been improved by Freire-Mane [FM] and Osserman-Sarnak [OS]. In both papers, the problem is reduced to the study of some Riccati type matrix equation of the form

$$
U^{\prime}(s)+U^{2}(s)+R(s)=0,
$$

where $R(s)$ is a symmetric matrix. The symmetry of $R(s)$ allows Green's results to be applied [Gr] to get estimates of the growth rate of Jacobi fields. In our case, the Riccati type equation deduced from (3) has the form

$$
U^{\prime}(s)+U^{2}(s)+A(s) U(s)+B(s)=0,
$$

where $B(s)$ is, in general, nonsymmetric and indefinite. In fact, a straightforward but lengthy calculation shows that $B(s)$ is symmetric and definite if and only if $F$ is totally geodesic, the sectional curvature of $M$ vanishes for all planes spanned by a vector tangent to $F$ and by a vector orthogonal to $F$, and the sectional curvature of the leaves has a constant sign. So, one could follow this procedure in some very special cases only and therefore estimating $h_{\mu}(\varphi)$ from below (for a smooth invariant measure $\mu$ ) seems to be promising.

(B) One may also ask: when is $\varphi$ Anosov? Some necessary conditions are obvious: $\operatorname{codim} F$ should be even and all geodesic flows of the leaves of $F$ should be uniformly hyperbolic, therefore the leaves could not admit conjugate points. Trying to study this problem by the methods analogous to those of $[E]$ we find the difficulty mentioned in (A): the matrix $B(s)$ in (24) need not be neither symmetric nor definite. It seems that the problem becomes even more interesting when $\varphi$ is the geodesic flow of a non-integrable subbundle of $T M$ (equipped with the connection induced from $T M$ by the orthogonal projection). In this case, $\varphi$ cannot be decomposed into the family of the geodesic flows of leaves.

(C) Let us note that there are several results concerning the existence of totally geodesic foliations of Riemannian manifolds. For example, it is known that codimension-one totally geodesic foliations of closed Riemannian manifolds of strictly positive (or, negative) Ricci (or, $k$-sectional) curvature do not exist [Br]. Also, there are no complete totally geodesic foliations of locally symmetric negatively curved Riemannian manifolds of finite volume [Z]. (A foliation of a Riemannian manifold is said to be complete when all its leaves are complete with respect to the induced metric.)

In this context several questions arise. For example, do totally geodesic foliations (of codimension greater than one) exist on compaet manifolds of negative $k$-sectional curvature? Or, when it is the set of regular points (in the sense of Oseledets' Ergodic Theorem) of $\varphi$ non-empty (if the foliated manifold is not compact)? Is this true when $M$ has negative curvature and finite volume, and $F$ is close to geodesic? 
An answer to these questions could allow us to reprove (or, to improve) Zeghib's result.

(D) In [GLW], the geometric entropy of a foliation of a compact Riemannian manifold is defined. It is shown that this entropy depends on the transverse structure of a foliation, more precisely, on its holonomy pseudogroup. Therefore, the authors expect that the topological entropy of the geodesic flow of a foliation $F$ could be related to the geometric entropy of $F$, the rate of growth of leaves and the second fundamental tensor of $F$.

\section{REFERENCES}

[A] D. V. Anosov. Geodesic flows on closed Riemannian manifolds with negative curvature. Trudy Mat. Inst. Steklov 90 (1967), 1-235 (in Russian, English translation: Proc. Steklov Inst. Math.).

[BBE] W. Ballman, M. Brin \& P. Eberlein. Structure of manifolds of non-positive curvature, I. Ann. of Math. 122 (1985), 171-203.

[BBS] W. Ballman, M. Brin \& R. Spatzier. Structure of manifolds of non-positive curvature, II. Ann. of Math. 122 (1985), 205-235.

[Br] F. G. B. Brito. Une obstruction géométrique a l'existence de feuilletages de codimension 1 totalement géodésiques. J. Diff. Geom. 16 (1981), 675-684.

[E] P. Eberlein. When is a geodesic flow of Anosov type? J. Diff. Geom. 8 (1972), I 437-463, II 565-577.

[FM] A. Freire \& R. Mañe. On the entropy of the geodesic flow in manifolds without conjugate points. Invent. Math. 69 (1982), 375-392.

[Ga] L. Garnett. Foliations, the ergodic theorem and Brownian motion. J. Func. Anal. 51 (1983), 285-311.

[GLW] E. Ghys, R. Langevin \& P. G. Walczak. Entropie géométrique des feuilletages. Acta Math. 168 (1988), 105-142.

[Gr] L. W. Green. A theorem of E. Hopf. Mich. Math. J. 5 (1958), 31-34.

[GKM] D. Gromoll, W. Klingenberg \& W. Meyer. Riemannsche Geometrie im Grossen, Springer Verlag: Berlin-Heidelberg-New York, 1968.

[KN] S. Kobayashi \& K. Nomizu. Foundations of Differential Geometry, II, Interscience Publishers: New York-London-Sydney, 1969.

[K] W. Klingenberg. Lecture on Closed Geodesics, Springer Verlag: Berlin-Heidelberg-New York, 1978.

[M] R. Mañe. Ergodic Theory and Differentiable Dynamics, Springer Verlag: Berlin-HeidelbergNew York, 1987.

[Ma1] A. Manning. Topological entropy for geodesic flows. Ann. of Math. 110 (1979), 567-573.

[Ma2] A. Manning. Curvature bounds for the entropy of the geodesic flow on a surface. J. London Math. Soc. 24 (1981), 351-357.

[O] V. I. Oseledets. A multiplicative ergodic theorem. Lyapunov characteristic numbers for dynamical systems. Trudy Moskov. Mat. Obshch. 19 (1968), 197-221 (in Russian, English translation: Trans. Moscow Math. Soc.).

[OS] R. Osserman \& P. Sarnak. A new curvature invariant and entropy of geodesic flows. Invent. Math. 77 (1984), 455-462.

[P] Ya. B. Pesin. Lyapunov characteristic exponents and smooth ergodic theory. Usp. Mat. Nauk 32 (1977), 55-111 (in Russian, English translation: Russ. Math. Surveys).

[PI] J. Plante. Foliations with measure preserving holonomy. Ann. of Math. 102 (1975), 327-361.

[R] D. Ruelle. Ergodic theory of differentiable dynamical systems. Publ. Math. IHES 50 (1979), 275-305

[S] P. Sarnak. Entropy estimates for geodesic flows. Ergod. Th. \& Dynam. Sys. 2 (1982), 513-524.

[Wa] P. Walters. An Introduction to Ergodic Theory, Springer Verlag: Berlin-Heidelberg-New York, 1982.

[Z] A. Zeghib. Feuilletages géodésiques des variétés localement symétriques et applications. Thèse, Univ. de Dijon, 1985. 